\title{
Technique
}

\section{New device for progressive dilatation of benign oesophageal stricture}

P. A. MAZURE, J. C. CHIOCCA, and A. G. SFERCO From the Gastroenterology Service, Policlínico Professor Alejandro Posadas Haedo, Buenos Aires, Argentina

When a $2 \mathrm{~mm}$ OD polyethylene tube is left within a strictured oesophagus for feeding purposes some dilatation of the stricture occurs. Based on this observation, we have threaded a $4 \mathrm{~mm}$ OD tube (and a $6 \mathrm{~mm}$ OD tube thereafter) over the original $2 \mathrm{~mm}$ tube and left it within the oesophageal stricture for $\mathbf{4 8}$ hours. This method is inconvenient because tubes beyond $6 \mathrm{~mm}$ OD irritate the hypopharynx. We therefore designed the following technique.

\section{Design}

A plastic radiopaque sheath $10 \mathrm{~cm}$ long is cemented at $6 \mathrm{~cm}$ from the distal end of a pliable, soft, radiopaque polyethylene nasogastric tube $50 \mathrm{~cm}$ long and $5 \mathrm{~mm}$ OD. A series of these dilators was constructed so that the outer plastic sheath ranged in diameter from 8 to $16 \mathrm{~mm}$ in $2 \mathrm{~mm}$ steps. A plastic-tipped $4 \mathrm{~mm}$ Jackson bougie slides easily into the nasogastric tube, and is used to give enough rigidity to insert the tube into the oesophagus (Fig. 1).

Received for publication 7 December 1971.

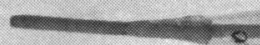

Fig. Close-up picture of the plastic dilator. Note the holes drilled at both ends.

\section{Technique}

After the $6 \mathrm{~mm}$ diameter tube has been removed, the plastic dilator is introduced into the oesophagus under $x$-ray control with the Jackson bougie inserted in the nasogastric tube. Once the tip of the latter reaches the stomach and the plastic sheath is located within the stricture the bougie is removed and the patient is fed through the tube with a liquid diet. We usually begin with the $8 \mathrm{~mm}$ OD sheath, leaving it within the stricture for 48 hours. The dilator is then changed for the following size every 48 hours until we reach the $16 \mathrm{~mm}$ diameter. In general the patient tolerates the dilator well, and we feed him through the nasogastric tube until the diameter of the oesophageal lumen is restored to a size at which a liquid or a semisolid diet can be re-established. This takes about two to three weeks.

Most of the patients produce excessive amounts of saliva, which could not be swallowed because the plastic sheath totally occluded the lumen of the oesophagus at the level of the stricture. This problem was solved by drilling holes at the upper and lower ends of the plastic sheath which allows saliva to reach the stomach and the patient to take sips of water.

\section{Results}

We have attempted this method of dilatation in five patients seen in the last two years. Four had reflux oesophagitis, the fifth had a stricture due to an alleged oesophagitis secondary to tetanus antitoxin administration. Up to date the four patients with reflux oesophagitis have no dysphagia, and the calibre of the oesophagii as measured radiographically is greater than $16 \mathrm{~mm}$ in all of them. The fifth patient improved with the treatment, but was lost to follow up. There were no failures with the intubation procedure. 